\title{
Parthénocarpie naturelle chez la tomate. I. - Revue bibliographique
}

\author{
Jacqueline PHILOUZE
}

I.N.R.A., Station d'Amélioration des Plantes maraîchères, Centre de Recherches d'Avignon, B.P. 94, F 84140 Montfavet

La parthénocarpie, définie comme la production de fruits sans graines, peut être naturelle ou artificielle. Dans une $1^{\text {rc }}$ partie, sont donnés quelques exemples de parthénocarpie artificielle chez la tomate, Lycopersicon esculentum Mill., de façon à mieux faire la distinction avec la parthénocarpie naturelle, qui fait l'objet de cette étude. Plusieurs cas de parthénocarpie naturelle épars dans la littérature sont cités.

Dans une $2^{c}$ partic, les travaux réalisés par 4 équipes de chercheurs dans des pays différents sont présentés de façon détaillée :

1) en Allemagne fédérale, travaux avec un matériel génétique diversifié dont le plus intéressant est celui issu du croisement «Atom » $x$ «Bubjekosoko »;

2) en Italie, travaux avec un matériel présentant une aptitude à la parthénocarpie due au gène récessif pat ;

3) en Moldavie soviétique, travaux avec la variété « Pridneprovskij";

4) en Russie soviétique, travaux avec la variété « Severianin » dont l'aptitude à la parthénocarpie est due au gène récessif pat-2.

Des études comparatives ont été faites entre, d'une part, des fruits sans graines d'une variété parthénocarpique et, d'autre part, des fruits avec graines, soit de la même variété, soit de variétés non parthénocarpiques, pour les caractères agronomiques, physiologiques, biochimiques. Des études ont également été faites sur les facteurs du milieu favorables à l'expression de la parthénocarpie, sur la teneur des ovaires en substances de croissance, etc...

Les résultats obtenus par ces 4 équipes ont stimulé les recherches dans plusieurs pays, dont la France. La prospection de nouveau matériel parthénocarpique a été entreprise, en particulier dans le matériel issu de croisements interspécifiques.

Les exemples connus de parthénocarpie naturelle chez la tomate peuvent être rattachés à 2 grands groupes : parthénocarpie accidentelle ou parthénocarpie facultative, cette dernière étant de beaucoup la plus intéressante.

Mots clés additionnels : Parthénocarpie accidentelle, parthénocarpie facultative, Lycopersicon esculentum.

\section{Natural parthenocarpy in tomato. I. Review of bibliography}

Parthenocarpy, defined as the production of fruits without seeds, may be natural or artificial. In the first part, some examples of artificial parthenocarpy in tomato, Lycopersicon esculentum Mill., are given, to make clearer the distinction with natural parthenocarpy, which is studied in this paper. Several examples of natural parthenocarpy scattered in the literature are mentioned.

In the second part, the work of 4 teams of researchers in different countries is presented in more detail :

1) in West Germany, work with diversified genetic material, the most interesting originating from the cross "Atom" $\times$ "Bubjekosoko";

2) in Italy, work with material, in which parthenocarpy is due to the recessive gene pat;

3) in Soviet Moldavia, work with the variety 'Pridneprovskij';

4) in Soviet Russia, work with the variety "Severyanin ", in which parthenocarpy is due to the recessive gene pat 2.

Comparative studies have been made between seedless fruits of a parthenocarpic variety and seeded fruits, either of the same variety, or of non-parthenocarpic varieties, for agronomic, physiological, and biochemical characters. Some studies have also been done on environmental factors favorable to the expression of parthenocarpy, on auxin content of ovaries, etc...

The results obtained by these 4 teams have stimulated research in several countries, in particular in France. A search has been made for new parthenocarpic material, in particular in progeny from interspecific crosses. The known examples of natural parthenocarpy in tomato may be classified in 2 main groups : accidental parthenocarpy or facultative parthenocarpy, the latter being much more interesting.

Additional key words : Accidental parthenocarpy, facultative parthenocarpy, Lycopersicon esculentum. 


\section{INTRODUCTION}

En guise d'introduction, nous donnerons quelques définitions concernant la parthénocarpie et valables pour l'ensemble du règne végétal.

Une importante revue bibliographique sur la parthénocarpie a été faite par GUSTAFSON en 1942 ; cet auteur cite des exemples de parthénocarpie naturelle ou artificielle chez environ 70 genres différents.

Le terme de parthénocarpie a été introduit par NOLL en 1902 (cité par GUSTAFSON, 1942), pour définir la formation de fruits sans graines, résultant de l'absence de pollinisation ou de toute autre stimulation. WINKLER, en 1908 (cité par GUSTAFSON, 1942) définit la parthénocarpie comme la production de fruits sans graines ou avec des graines vides ; il distingue entre parthénocarpie stimulative, dans le cas où le fruit est produit après une pollinisation ou une autre stimulation, et parthénocarpie végétative, chez laquelle ni pollinisation ni aucune autre stimulation n'est nécessaire. GUSTAFSON (1942) utilise le terme de parthénocarpie pour le développement d'un fruit sans fécondation. Dans le cas où il $\mathrm{y} \mathrm{a}$ eu fécondation avec avortement précoce de l'embryon, STCUT (1936) (cité par GuSTAFSON, 1942) emploie le terme de sténospermocarpie, ou fausse parthénocarpie. En fait, dans la pratique, il est très difficile de distinguer entre absence de fécondation et avortement précoce de l'embryon (GUSTAFSON, 1942).

GUSTAFSON (1942) classe les cas de parthénocarpie en 2 grands groupes : parthénocarpie naturelle et parthénocarpie artificielle induite expérimentalement par des substances chimiques. La tendance à la parthénocarpie naturelle peut être améliorée en créant artificiellement des conditions de milieu favorables à l'expression de cette parthénocarpie ; il est donc parfois difficile de dire où finit la parthénocarpie naturelle et où commence la parthénocarpie artificielle ou induite.

NITSCH (1952) distingue entre parthénocarpie génétique, parthénocarpie due aux facteurs du milieu et parthénocarpie d'origine chimique. Dans le cas de la parthénocarpie due aux facteurs du milieu, il s'agit de la formation de fruits parthénocarpiques due à des conditions inhabituelles du climat chez des plantes qui produisent d'habitude des fruits avec graines.

D'après ULRICH (1952), la parthénocarpie naturelle est tantôt constante et caractéristique de la variété, tantôt facultative, tantòt accidentelle.

VAZART (1955), en faisant la synthèse des définitions données par les auteurs précédents, a proposé une classification des différents types de parthénocarpie, qui peut être naturelle ou artificielle. La parthénocarpie naturelle peut être obligatoire (d'origine génétique), facultative ou accidentelle (dans ces 2 derniers cas, elle serait due à des modifications des conditions habituelles, ou bien son origine serait indéterminée).

GUSTAFSON (1939) a montré le rôle des auxines dans la formation des fruits naturellement parthénocarpiques. Chez ces fruits, la teneur en auxines dans les ovaires des boutons floraux est plus élevée que celle des ovaires des boutons floraux de variétés comparables non parthénocarpiques (essais faits sur des variétés d'oranger, de citronnier et de vigne). Le taux d'auxines serait suffisant chez les variétés parthénocarpiques pour que l'ovaire se développe sans pollinisation ni fécondation. Pour NITSCH (1970), l'important pour une plante parthénocarpique est d'arriver à une concentration seuil en substances de croissance au moment critique de l'anthèse. Rappelons en effet que chez un fruit non parthénocarpique, les différentes étapes du développement du fruit sont à mettre en relation avec sa teneur en auxines (NITSCH, 1952) :

1) Le développement de l'ovaire commence au stade très jeune bouton floral et se poursuit régulièrement jusqu'à l'anthèse, puis s'arrête brusquement, à moins que les ovules ne soient fécondés. Ceci correspond à une chute de la teneur de l'ovaire en auxines, teneur qui devient presque nulle au moment où se produit normalement la pollinisation. A ce stade, une fleur non pollinisée tombera le plus souvent.

2) Au moment de la pollinisation, il y a reprise du développement $\mathrm{du}$ fruit, le pollen et les tubes polliniques agissant soit directement par les auxines qu'ils contiennent, soit indirectement par l'induction de la synthèse d'auxines par le pistil, grâce aux enzymes qu'ils contiennent.

3) Le fruit poursuit sa croissance grâce aux auxines produites par les graines qui se développent, auxines qui diffusent dans l'ovaire. Chez la tomate, il est ainsi bien connu que, chez une même variété, il y a une corrélation entre le poids des fruits et leur nombre de graines.

Dans cet article, nous présenterons un certain nombre d'exemples, épars dans la littérature, de parthénocarpie chez la tomate cultivée, Lycopersicon esculentum Mill. Dans une $2^{\mathrm{e}}$ partie, nous présenterons de façon beaucoup plus complète le travail réalisé sur la parthénocarpie naturelle chez la tomate par plusieurs équipes de chercheurs avec du matériel végétal et dans des conditions extrêmement différentes ; ce travail ouvre des perspectives intéressantes pour les généticiens, les sélectionneurs et les physiologistes.

\section{QUELQUES EXEMPLES DE PARTHÉNOCARPIE CHEZ LA TOMATE}

Nous retiendrons, de la terminologie utilisée dans l'introduction, que la parthénocarpie est le développement d'un fruit sans fécondation, et que cette parthénocarpie peut être naturelle ou artificielle. La parthénocarpie artificielle n'est pas ce qui nous intéresse ici, nous en donnerons cependant quelques exemples, afin de mieux faire la distinction avec la parthénocarpie naturelle.

\section{A. Parthénocarpie artificielle}

Elle peut être induite par toutes sortes de traitements, qui vont de l'application, sur le stigmate, de pollen d'espèces ou de genres différents, de pollen irradié, de pollen mort, d'extraits de pollen, jusqu'à la pulvérisation de substances de croissance.

Ainsi TATARINTSEV (1929) a traité le stigmate de fleurs castrées de plusieurs variétés de différentes façons : apport de pollen de pomme de terre, de spores de lycopode, de terre séchée, stigmate irrité avec une pince ou avec un pinceau. Dans ces conditions, 60 à 70 p. 100 des fleurs de «Ficarazzi » donnaient des fruits parthénocarpiques, alors que chez le témoin (fleurs castrées sans traitement du stigmate), 10 p. 100 des fleurs donnaient des fruits. Les autres variétés testées répondaient également aux différents traitements par la formation de fruits parthénocarpiques, quoique dans des proportions différentes de celles de «Ficarazzi ». Les fruits parthénocarpiques diffèrent le plus souvent des fruits avec graines par leur taille, leur forme et leur structure interne.

GUSTAFSON (1936) a été le premier à induire le développement de fruits parthénocarpiques chez la tomate en utilisant des substances chimiques : acides indole-propioni- 
que, phénylacétique, indole-acétique, indole-butyrique. Depuis, de très nombreuses substances chimiques ont été utilisées avec succès (citons en particulier l'acide naphtoxyacétique). La pulvérisation de substances de croissance est maintenant utilisée de façon courante par de nombreux agriculteurs, en particulier sur les premières inflorescences en culture précoce, de façon à pallier une insuffisance de la pollinisation.

\section{B. Parthénocarpie naturelle}

Les plantes mâle-stériles, ou présentant des anomalies du nombre de chromosomes, montrent souvent une certaine aptitude à développer des fruits parthénocarpiques. Ainsi LESLEY \& LESLEY (1941) ont montré que la déficience d'une partic d'un chromosome, ou que l'addition de ce chromosome, déficient ou entier, entraînait une certaine tendance à la parthénocarpie; les fruits sans graines, comparés à ceux avec graines obtenus sur la même plante, étaient beaucoup plus petits et plus lents à mûrir. Nous laisserons de côté tous les cas de ce genre, aneuploïdie ou polyploïdic, pour ne nous intéresser qu'à la parthénocarpie rencontrée chez des plantes diploïdes normales.

Plusieurs auteurs ont montré que les conditions de milieu jouent un rôle important dans la production naturelle de fruits parthénocarpiques. La température (élevée ou basse) est le plus souvent citée, mais aussi la lumière (intensité lumineuse, photopériode), la fumure, etc... Il est difficile de savoir à quel niveau jouent d'abord les conditions de milieu. Favorisent-elles l'expression d'une parthénocarpie naturelle latente en entravant les processus normaux de formation du pollen, de pollinisation et de fécondation, ou bien agissentelles en stimulant la formation de substances de croissance dans les ovaires des jeunes boutons floraux, ou enfin ces 2 phénomènes se superposent-ils ?

HÖSTERMANN (1912-1913) (cité par GuSTAFSON, 1942) a castré des fleurs de plusieurs variétés de tomate à plusieurs reprises en été, afin de déceler celles qui avaient une tendance naturelle à la parthénocarpie. Aucune des variétés n'a développé régulièrement des fruits parthénocarpiques, mais toutes en ont produit occasionnellement, le plus souvent de petite taille, surtout en fin de saison.

D'après HAWTHORN (1937), des lignées issues du croisement entre «Large Cherry » et «Bonny Best » sont capables de nouer au Texas pendant les mois chauds; il y a production de fruits avec graines en juin et en début juillet, puis de fruits sans graines quand les températures sont très élevées et, à nouveau, de fruits avec graines en novembre.

Pour FosTer \& TATMAN (1937) (cité par GUSTAFSON, 1942), les températures élevées ou basses, les réserves faibles en hydrates de carbone et en azote sont des causes de la production de fruits parthénocarpiques. Les réserves faibles en hydrates de carbone sont causées par une fumure azotée élevée, une humidité élevée du sol, une température élevée et des jours courts.

Pour OsBorne \& WENT (1953), des fleurs castrées de la variété «Essex Wonder» ne développent de petits fruits parthénocarpiques que dans des conditions de températures basses, d'intensité lumineuse élevée et de photopériode longue; dans ces conditions, les réserves en hydrates de carbone sont importantes. Des réserves faibles en hydrates de carbone induisent la stérilité et non la parthénocarpie chez « Essex Wonder », contrairement à ce que suggèrent FOSTER \& TATMAN (1937).

JOHNSON \& HALL (1954) ont montré que, en culture d'été au Texas, « Marglobe " et « Rutgers » ne produisaient pas de fruits. D’autres variétés («Porter», «Summer Prolific » et 5 lignées sélectionnées au Texas) produisaient de nombreux fruits parthénocarpiques. Il y avait des différences marquées dans l'aptitude à former des fruits avec ou sans graines à températures élevées dans ce matériel. Les auteurs concluent qu'il doit être possible de sélectionner pour un degré élevé de parthénocarpie.

Les variétés «Oregon Cherry » (BAGGETT \& FrAZIER, 1978a) et surtout «Oregon T 5-4 » (BAGGETT \& FRAZIER, 1978b), toutes 2 à très petits fruits (11 à $16 \mathrm{~g}$ ), montrent une tendance à former des fruits parthénocarpiques à température basse. Les fruits sans graines de « Oregon T 5-4 » auraient une maturation plus rapide que les fruits avec graines.

El AHMAdi \& STEVEns (1979) ont montré que, chez la lignée «BL $6807=$ Sub Arctic Plenty », sélectionnée dans l'Alberta pour son aptitude à nouer au froid (HARRIS, 1972), 75 p. 100 des fruits formés à températures élevées ne contenaient pas de graines (essai en chambre climatisée à $37^{\circ} 8$ le jour, $26^{\circ} 7$ la nuit, photopériode de $12 \mathrm{~h}$ ).

\section{TRAVAUX RÉALISÉS SUR LA PARTHÉNOCAR- PIE NATURELLE CHEZ LA TOMATE}

Des travaux ont été conduits par des équipes différentes, dans des pays différents, avec du matériel végétal, des méthodes d'étude et des objectifs différents. Faire une synthèse à partir d'éléments aussi disparates serait bien difficile, aussi avons-nous choisi de présenter séparément les travaux conduits initialement par 4 équipes de chercheurs, en Allemagne fédérale, en Italie, en Moldavie soviétique et en Russie soviétique. Les résultats obtenus par ces équipes ont ouvert la voie à des recherches conduites maintenant dans plusieurs pays.

\section{A. Matériel dont l'intérêt a été mis en évidence en Allemagne fédérale}

\section{Travaux entrepris en Allemagne fédérale}

REIMANN-PHILIPP (1968) a signalé la sélection de matériel parthénocarpique au Max-Planck-Institut für Kulturpflanzenzüchtung, à Ahrensburg. Ce matériel a ensuite été étudié au Bundesforschungsanstalt für Gartenbauliche Pflanzenzüchtung, à Ahrensburg.

Le matériel étudié, assez diversifié, est issu de plusieurs croisements différents, aussi présentons-nous dans le tableau 1 la généalogie des variétés que nous mentionnons ici, généalogie qui nous a été communiquée par REIMANNPHILIPP (comm. pers., 1977).

Le matériel parthénocarpique sélectionné au MaxPlanck-Institut est intéressant pour sa très grande précocité, $\mathrm{du}$ fait de l'amélioration de la nouaison des premiers bouquets quand les conditions climatiques sont défavorables (REIMANN-PHILIPP, 1968). En culture précoce, il y a d'abord production de fruits sans graines, puis les fruits contiennent des graines (REIMANN-PHILIPP \& FuCHS, 1969). Cette aptitude à la parthénocarpie est héréditaire (REIMANN-PHILIPP \& FUCHS, 1969). Mais ce matériel parthénocarpique, tout comme le matériel non parthénocarpique, exige un éclairage d'appoint en hiver (REIMANNPHILIPP, 1968 ; REIMANN-PHILIPP \& FuCHS, 1969).

Preil \& ReIMANN-Philipp (1969) ont montré que la parthénocarpie de 5 lignées étudiées: « 65/107», «67/235», «66/111-1», «66/111-2» et «66/125-2», ne vient pas d'un défaut de qualité du pollen, qui est très fertile même à basse température. Des fruits parthénocarpiques 
TABLEAU 1

Généalogie du matériel parthénocarpique originaire d'Allemagne fédérale (d'après REIMANN-PHILIPP, comm. pers., 1977). Pedigree of the parthenocarpic material from West Germany (after REIMANN-PHILIPP, pers. comm., 1977).

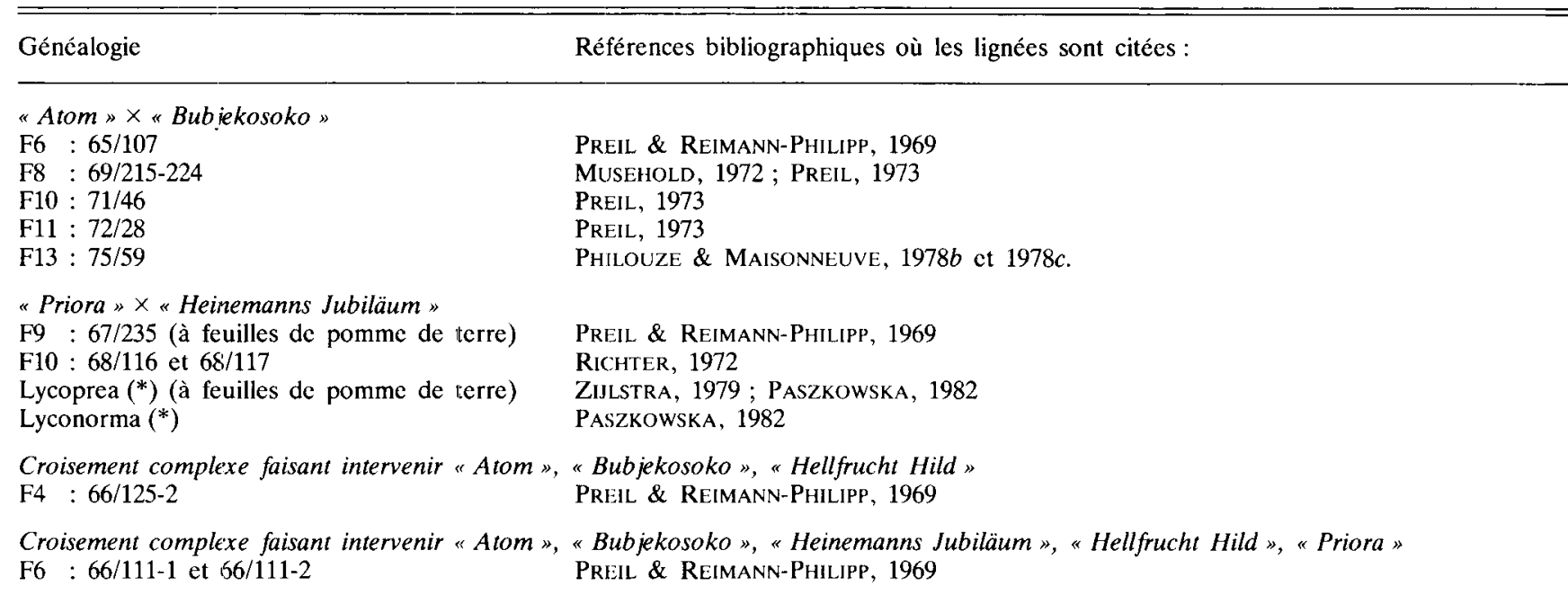

(*) «Lycoprea » et «Lyconorma » sont inscrites au Catalogue Officicl des variétés en Allemagne fédérale.

peuvent se former bien que des tubes polliniques atteignent l'ovaire. Ces auteurs font l'hypothèse d'une concentration élevée en substances de croissance dans l'ovaire qui provoquerait le grossissement de l'ovaire avant que la fécondation ne se produise.

MUSEHOLD (1972) a étudié la relation entre la variation spontanéc du niveau de parthénocarpie et la teneur en substances de croissance chez « 69/215-224 » (F8 du croisement «Atom » $x$ «Bubjekosoko ») en comparaison avec 2 lignées ne donnant pas de fruits parthénocarpiques. La teneur des ovaires en substances de croissance liées ne semble pas avoir d'influence sur la parthénocarpic. Chez «69/215-224 », les ovaires qui donnent des fruits parthénocarpiques ont une teneur élevée en substances de croissance libres; les variations de parthćnocarpie sont aussi abruptes que la teneur des ovaires en substances de croissance libres. $\mathrm{Au}$ début de la floraison, les lignées non parthénocarpiques contiennent beaucoup moins de substances de croissance libres que «69/215-224"; cependant la teneur maximale en substances de croissance libres des lignées non parthénocarpiques n'est pas significativement différente de celle de «69/215-224». MUSEHOLD fait l'hypothèse d'un facteur génétique qui permettrait l'action des substances de croissance chez "69/215-224» ou qui la bloquerait chez le matériel non parthénocarpique. MUSEHOLD (1972) et PREIL (1973) expliquent ainsi la formation de fruits parthénocarpiques : pendant la formation des fleurs, des facteurs externes stimulent la production de substances de croissance, de telle sorte que l'ovaire commence à grossir avant que la fécondation soit possible, ce qui explique que, au moment où la fécondation devrait normalement se produire, celle-ci soit devenue impossible par suite de la dégénérescence des ovules; il en résulte la formation de fruits parthénocarpiques. A la suite de changements dans les conditions climatiques, la teneur en substances de croissance des jeunes ovaires diminue, la fécondation redevient possible ; il y a alors production d'un pourcentage variable de fruits contenant des graines.
PreIL (1973) a montré le rôle de la température sur la formation de fruits parthénocarpiques. Les essais ont été conduits sur des lignées issues de croisements différents. Ce sont les lignées «69/215-224», «71/46», «72/28», issues du croisement «Atom» $x$ «Bubjekosoko», qui montrent la plus forte proportion de fruits parthénocarpiques. Les essais conduits pendant 3 ans au champ ont montré une concordance entre les minima des courbes des températures journalières moyennes (faites sur la base de relevés horaires) et les maxima des courbes des pourcentages de fruits parthénocarpiques, avec un décalage dans le temps de 62 à 68 jours. L'effet des températures basses sur la formation de fruits parthénocarpiques a été vérifié en chambre climatiséc sous intensité lumineuse de 30000 lux, à une température diurne de $25^{\circ} \mathrm{C}$ et des températures nocturnes de $5^{\circ}, 10^{\circ}$ et $15^{\circ} \mathrm{C}$. Les pourcentages de fruits parthénocarpiques ont été respectivement 71 p. 100,45 p. 100 et 29 p. 100 . Cependant, selon PREIL, la température n'est probablement pas le seul facteur important, la lumière (longueur du jour, intensité lumincuse, composition de la lumière) et les interactions lumière $\times$ température jouent certainement un rôle.

RICHTER (1972) a comparé la structure et la composition chimique des fruits sans graines et des fruits avec graines de 2 lignées parthénocarpiques, «68/116» ct «68/117》. Les fruits sans graines ont une teneur en matière sèche plus élevée, que l'on considère le fruit entier (graines comprises quand il y en a), ou les différentes parties du fruit (péricarpe, parois des loges et placentas, pulpe); leur teneur en acides est plus faible. Par contre, RICHTER n'a pas trouvé de différences qualitatives dans les substances aromatiques entre fruits sans graines et fruits avec graines d'une même lignée.

Le travail entrepris à Ahrensburg sur la parthénocarpie chez la tomate, est maintenant considéré comme terminé (PREIL, 1978) : la culture précoce sous serre de cette espèce n'est en effet plus économiquement rentable dans les conditions allemandes depuis la crise de l'énergie. 


\section{Travaux entrepris dans d'autres pays}

En France, pour PhILOUZE \& MAISONNEUVE (1978b; 1978 c), l'aptitude à la parthénocarpie de «75/59 » serait sous le contrôle d'au moins 3 gènes récessifs. Ces auteurs ont montré (1978a) que les fruits sans graines de « 75/59», obtenus à partir de fleurs castrées et non pollinisées, sont de même grosseur que les fruits avec graines obtenus après castration et autofécondation ou après pollinisation avec le pollen d'une autre variété, quelle que soit la période de l'année.

La variété «Lycoprea " a été testée aux Pays-Bas (ZIJLSTRA, 1979). Après castration des fleurs sans fécondation, l'aptitude à la parthénocarpie s'est montrée très faible ; les fruits sans graines étaient 2 fois moins lourds que les fruits avec graines.

Pour PASZKowsKa (1982), en Pologne, l'aptitude à la parthénocarpie de "Lyconorma " et "Lycoprea » serait monogénique récessive.

\section{B. Matériel dont l'intérêt a été mis en évidence en Italie}

\section{Travaux réalisés en Italie}

BIANCHI \& SORESSI (1969), SORESSI (1970) ont décrit un mutant à anthères courtes obtenu dans la variété « Roma » après traitements de graines au méthane sulfonate d'éthyle ; le symbole sha (short anthers) a été attribué à l'allèle responsable de ce caractère. Ce mutant est caractérisé par une semi-stérilité et par un pourcentage élevé de fruits parthénocarpiques. Ce $2^{\mathrm{e}}$ caractère, pris tout d'abord pour un effet pléiotropique d'un seul gène récessif, a par la suite été attribué à un $2^{\mathrm{c}}$ gène récessif, très étroitement lié à sha, responsable du développement précoce de l'ovaire sans pollinisation et sans fécondation. Le symbole proposé est pat (parthenocarpic fruit) (SORESSI \& SALAMINI, 1975).

Ce mutant a été étudié sous l'angle génétique et agronomique à la Station de Montanaso Lombardo (Milan) dépendant de l'Istituto Sperimentale per l'Orticoltura de Salerne. Des études biochimiques ont été conduites au Laboratorio Biosintesi Vegetale du C.N.R. (Consiglio Nazionale delle Ricerche), à Milan.

FALAVIGNA ét al. (1978) pensent à l'intérêt du caractère pat pour les variétés d'industrie. Dans des descendances F2, les plantes parthénocarpiques se montrent intéressantes pour leur précocité ; leurs fruits sont beaucoup plus petits que ceux des plantes non parthénocarpiques, mais le nombre de fruits est plus élevé. De plus, la teneur en sucres solubles des fruits des plantes parthénocarpiques est en moyenne un degré Brix supérieur à celui des fruits des plantes non parthénocarpiques.

Pour AllaVENA \& SORESSI (1978), l'association entre la parthénocarpie due au gène pat et la tétraploïdie devrait permettre de surmonter l'insuffisance de mise à fruit qui est de règle chez des tétraploïdes non parthénocarpiques.

MAPELLI et al. (1978) ont comparé la croissance du fruit de la variété «Ventura » et de sa version (sha pat). Chez la variété parthénocarpique, la croissance des ovaires est beaucoup plus rapide, mais à maturité ( $10 \mathrm{j}$ plus précoce que chez la variété non parthénocarpique), les fruits n'ont que les $2 / 3$ de la taille des fruits de la variété témoin non parthénocarpique. A l'anthèse, la concentration en auxine cst environ 3 fois plus élevée dans les ovaires parthénocarpiques que dans les ovaires non parthénocarpiques. L'évolution de la teneur en auxine et en gibbérelline des fruits parthénocarpiques et des fruits avec graines a été étudiée, de l'anthèse à $45 \mathrm{j}$ après l'anthèse, par MAPELLI et al.
(1978). MAPELLI (1981) a suivi de la même façon l'évolution de la teneur des fruits en cytokinine.

MAPELLI et al. (1979) ont comparé la production de graines de plantes F2 (sha pat) à celle de plantes (sha+ pat+) en serre, après fécondation libre ou à la suite d'une autofécondation manuelle; les plantes (sha pat), qui donnaient 98 p. 100 de fruits sans graines en fécondation libre, n'en donnaient plus que 17 p. 100 après pollinisation manuelle et, avec ce dernier traitement, il y avait en moyenne 42,3 graines par fruit contenant au moins une graine, tandis que, à la suite du même traitement, les fruits des plantes $(s h a+p a t+)$ en contenaient en moyenne 49,9 . MAPELLI et al. concluent que la parthénocarpie n'est pas due à une stérilité ou à une auto-incompatibilité. Cependant, la pollinisation manuelle doit intervenir 2 ou $3 \mathrm{j}$ avant l'anthèse ; plus tard, la pollinisation produit rarement des graines. Ceci semble être en relation avec la croissance rapide caractéristique de l'ovaire (sha pat) à l'anthèse. La pulvérisation d'acide gibbérellique effectuée sur des plantes de la version (sha pat) de "Ventura " favorise le développement des étamines, mais il n'y a pas induction de fruits avec graines.

\section{Travaux entrepris dans d'autres pays}

En France, les observations réalisées par PECAUT \& PHILOUZE (1978) ont conduit à des conclusions différentes de celles des auteurs italiens. D'après PeCaut \& Philouze, sha et pat sont des mutations survenues à un seul et même locus: la présence d'étamines courtes et le développement de fruits parthénocarpiques seraient des effets pléiotropiques d'une même mutation. Ces caractères sont en effet réapparus simultanément par mutation naturelle dans une lignée en cours de sélection à Montfavet. L'hybride F1 entre la lignée mutante, «Montfavet 191 », et le matériel (sha pat) reçu de SORESSI, avait le phénotype (sha pat). La probabilité de 2 mutations à 2 loci différents chez le même individu est très faible. D'autre part, il n'a jamais été possible d'observer de recombinants (shapat+) ou (sha+ pat) sur un matériel il est vrai peu abondant. Enfin PeCaut \& Philouze ont observé que l'expression du caractère «short anthers» dépendait du génotype et du milieu, au point d'avoir un aspect quasi normal dans certaines conditions. Ces auteurs ont par ailleurs noté que, si la fertilité du pollen de ce mutant était normale, la production de graines était extrêmement faible, quelle que soit l'origine du pollen utilisé.

Aux Pays-Bas, Hogenboom (1978, 1980, 1981, 1982) et ZIJLSTRA (1979) ont observé le mutant (sha pat), mais ces auteurs n'apportent aucune information nouvelle sur ce matériel.

\section{Matériel dont l'intérêt a été mis en évidence en Moldavie soviétique}

\section{Travaux entrepris en Moldavie soviétique}

Les travaux ont été conduits au Jardin Botanique de l'Académie des Sciences de la République de Moldavie, à Kichinev, par LUDNIKOVA (1970) qui a consacré sa thèse à l'étude de la parthénocarpie chez la tomate.

LUDNIKOVA a étudié plusieurs variétés parmi lesquelles certaines avaient une tendance à former des fruits sans graines. La variété «Pridneprovskij » a été sélectionnée par KRAEVOJ à Kiev, en Ukraine, par croisement d'une variété de $L$. esculentum greffée sur pomme de terre, avec une origine de L. cerasiforme (KRAEVOJ, 1949). L'hybride «tomate» $X$ «Cyphomandra » $n^{\circ} 1641$ et l'hybride 
"Cyphomandra" $\times$ «tomate" $\mathrm{n}^{\circ} 1154$, ont été obtenus par LuNEVA (1957) (citée par LudNiKova, 1970) par la pollinisation d'une tomate L. esculentum greffée sur "Cyphomandra ", avec du pollen de «Cyphomandra ».

Nous donnons dans le tableau 2 les résultats d'expériences conduites par LuDNIKOVA (1970) pendant plusicurs années, après castration de fleurs non suivie de pollinisation, sur plusieurs inflorescences de plusieurs variétés :

- «Pridneprovskij » et les hybrides « Tomate $\times$ Cyphomandra » $\mathrm{n}^{\circ} 1641$ et «Cyphomandra $\times$ Tomate » $\mathrm{n}^{\circ} 1154$ peuvent former des fruits parthénocarpiques, contrairement à la variété témoin "Moldavskij rannij ".

- Les variétés capables de donner des fruits parthénocarpiques ne le font que sur les bouquets inférieurs, jamais sur les bouquets supérieurs ( $4^{\mathrm{e}}$ et $5^{\mathrm{e}}$ bouquets).

- Le pourcentage de fruits parthénocarpiques formés sur les bouquets inférieurs chez «Pridneprovskij» est variable selon les années. Ainsi en 1962, où le temps a été chaud et sec pendant la période de végétation, il y a eu moins de fruits avec graines qu'en 1963 et 1964, où le temps était plus humide et plus froid.

Ces résultats sont à rapprocher des observations faites à Kiev par KRAEvoJ (1949) et RYBCHENKo (1960), à Kichinev par LudNikova (1970), sur la variété "Pridneprovskij " cn conditions de fécondation libre. D'après KRAEvOJ (1949), «Pridneprovskij » se caractérise à Kiev par la formation de fruits sans graines aux bouquets inférieurs, alors que les fruits des étages moyens et supérieurs ont peu de graines. A Moscou et plus au Nord, tous les fruits sont parthénocarpiques. A Krasnodar et à Odessa, les fruits ont peu de graines aux étages inférieurs et un nombre presque normal de graines aux étages moyens et supérieurs. RYBCHENKO (1960) (cité par LUDNIKOVA, 1970) rapporte que chez «Pridneprovskij» la formation de plus ou moins de graines dans les fruits dépend des conditions climatiques. D'autre part, une humidité élevée du sol et une fumure abondante permettent une diminution de la quantité de graines des fruits de «Pridnoprovskij». A Kichinev (LudNIKova, 1970), en fécondation libre, «Pridneprovskij » a donné 10 p. 100 de fruits parthénocarpiques sur les premiers bouquets en 1962 , et environ 50 p. 100 en 1963 et 1964.

LuDnikova (1970) a montré que la parthénocarpic de "Pridneprovskij » se transmet aux descendances de croiscments faisant intervenir cette variété et des variétés non parthénocarpiques. Les hybrides F1 ne sont pas parthénocarpiques, le caractère est donc récessif. En F2, on retrouve lc caractère de parthénocarpie en association, sauf chez 2 plantes, avec une série de caractères morphologiques que l'on trouve chez «Pridneprovskij»: feuilles non découpées, cncore moins découpées et avec une surface foliaire plus importante aux étages inférieurs, où se forment les fruits sans graines, qu'aux ćtages supérieurs (LUDNIKova a montré que la teneur en substances de croissance des feuilles inféricures dc «Pridneprovskij » était plus élevée que celle des feuilles plus découpées des variétés non parthénocarpiques); croissance déterminée; fleurs des bouquets inférieurs avec des sépales soudés sur les $2 / 3$ de leur longueur.

Des études histochimiques (LuDNIKOVA, 1970) ont montré que les ovaires des bouquets inférieurs de «Pridneprovskij " à tous les stades observés (boutons, fleurs, fruits de 10 à $20 \mathrm{j}$ ) contiennent plus d'acide indole-acétique que les ovaires de la variété «Moldavskij rannij », non parthénocarpique. Ce fait est probablement à mettre en relation avec l'observation, faite par LUDNIKOVA, que, aux étages inféricurs, le grossissement des ovaires se produit avant l'ćclosion de la fleur, c'est-à-dire avant la pollinisation. L'ćtude a également porté, pour les mêmes stades de développement des fleurs et des jeunes fruits, sur la teneur en peroxydase, en acide ascorbique, en combinaisons sulhydriques et en amidon.

Des études embryologiques (RYBCHENKO, 1959, 1960, citć par LUDNIKOVA, 1970 ; LUDNIKOVA, 1970) ont montré que la quantité d'ovules fertiles dans les ovaires des bouquets inférieurs de "Pridneprovskij» est faible. La dégénérescence des ovules se produit pendant la macrosporogenèse, la macrogamétogenèse et après la formation des sacs embryonnaires. D'autre part, RYBCHENKO a montré que chcz «Pridneprovskij», le pollen des bouquets inférieurs ne se développe pas normalement et dégénère, puis le pollen se développe normalement sur les bouquets supéricurs. Ces observations sont en accord avec celles de LUDNIKOVA sur la croissance in vitro du pollen des bouquets inféricurs et des bouquets supéricurs de « Pridneprovskij».

Enfin, LudNikova (1970) a étudié la structure des fruits sans graines et avec graines de «Pridneprovskij »; la teneur en pulpe des fruits ayant peu ou pas de graines est inférieure à celle des fruits avec graines; le tissu placentaire est plus abondant. D'autre part, les chloroplastes ne sont pas

\section{TABLEAU 2}

Nombre de fleurs et de fruits et pourcentage de nouaison après castration des fleurs de quelques variétés (d'après LUDNIKOVA, 1970). Number of flowers and fruits and percentage of fruit-setting afier emasculation of the flowers of some varieties (afier LUDNIKOVA, 1970).

\begin{tabular}{|c|c|c|c|c|c|c|c|}
\hline \multirow{2}{*}{ Annćc } & \multirow{2}{*}{ Variété } & \multicolumn{3}{|c|}{ Sur les premicrs bouquets } & \multicolumn{3}{|c|}{ Sur les $4^{c}$ et $5^{c}$ bouquets } \\
\hline & & $\begin{array}{c}\text { Nbre fleurs } \\
\text { castrées }\end{array}$ & $\begin{array}{l}\text { Nbre } \\
\text { fruits }\end{array}$ & $\begin{array}{c}\% \\
\text { nouaison }\end{array}$ & $\begin{array}{c}\text { Nbre fleurs } \\
\text { castrées }\end{array}$ & $\begin{array}{l}\text { Nbre } \\
\text { fruits }\end{array}$ & $\begin{array}{c}\% \\
\text { nouaison }\end{array}$ \\
\hline 1962 & Pridncprovskij & 96 & 6 & 6 & - & - & - \\
\hline 1963 & $\begin{array}{l}\text { Pridneprovskij } \\
\text { Hybride «Tomate } \times \text { Cyphomandra } " n^{\circ} 1641 \\
\text { Hybride «Cyphomandra } \times \text { Tomate } " n^{\circ} 1154 \\
\text { Moldavskij rannij }\end{array}$ & $\begin{array}{l}36 \\
25 \\
40 \\
41\end{array}$ & $\begin{array}{r}18 \\
6 \\
2 \\
0\end{array}$ & $\begin{array}{r}50 \\
24 \\
5 \\
0\end{array}$ & $\begin{array}{l}35 \\
29 \\
36 \\
27\end{array}$ & $\begin{array}{l}0 \\
0 \\
0 \\
0\end{array}$ & $\begin{array}{l}0 \\
0 \\
0 \\
0\end{array}$ \\
\hline 1964 & Pridneprovskij & 276 & 69 & 25 & - & - & - \\
\hline
\end{tabular}


toujours détruits et on peut trouver une pulpe restée verte dans des fruits mûrs, dans certains cas.

Le travail réalisé par LUDNIKOVA n'a pas été poursuivi en Moldavie soviétique.

\section{Travaux entrepris dans d'autres pays}

Nous ne connaissons pas de travaux réalisés avec lc matériel parthénocarpique étudié par LUDNIKOVA.

\section{Matériel dont l'intérêt a été mis en évidence en Russie soviétique}

\section{Travaux entrepris en Russie soviétique}

Ces travaux concernent la variété «Severianin», sélectionnée par SolovjEVA à la Station expérimentale de Gribovo, près de Moscou. Cette variété est issue du croisement [«Byzon» greffé sur Solanum muricatum Ait. $\times$ F1 («Gruntovij Gribovskij» $\times$ L. hirsutum)] (IGNA. TOVA, comm. pers., 1977). Les travaux ont été conduits à l'Académic Timiriazev à Moscou, par un étudiant égyptien, DOVEDAR, qui a présenté sa thèse en 1973 sur les problèmes de fructification des tomates à température élevée. DOVEDAR a, d'une part, recherché des variétés capables de produire du pollen de bonne qualité à température élevée, il s'est, d'autre part, intéressé à «Severianin » capable de produire naturellement des fruits parthénocarpiques de même grosseur que les fruits avec graines, dans des conditions difficiles $\left(25^{\circ}-35^{\circ} \mathrm{C}\right.$ le jour, $20^{\circ}-25^{\circ} \mathrm{C}$ la nuit).

DOVEDAR (1973) a comparé chez "Severianin " le pourcentage de fruits parthénocarpiques, le poids des fruits avec et sans graines, le nombre moyen de graines par fruit, à la suite de 4 traitements:

- témoin (fécondation libre)

- fleurs castrées non pollinisées

- flcurs castrées pollinisées avec du pollen viable de la variété «Belij Naliv 241 »

- fleurs castrées pollinisées avec du pollen non viable de «Belij Naliv 241 ».

Les résultats sont donnés dans le tableau 3.

Ce tableau montre que la variété «Severianin» peut former des fruits parthénocarpiques quand il y a castration des fleurs non suivie de pollinisation ou suivic d'une pollinisation avec du pollen non viable. Cependant, dans ces 2 cas, le pourcentage de nouaison est inférieur à celui du témoin (fécondation libre). Le pourcentage de nouaison est amélioré si on pollinise les fleurs castrées avec du pollen viable de «Belij Naliv 241 ». En fécondation libre, «Severianin » donne des fruits avec graines et des fruits sans graines qui sont au moins aussi gros que les fruits avec graines; les fruits avec graines contiennent autant de graines que les fruits issus de fleurs castrées et pollinisées avec du pollen de "Belij Naliv 241 ».

Pour savoir si la parthénocarpie chez «Severianin » est stimulative ou non stimulative (les traitements dont les résultats sont reportés dans le tableau 3 ne permettent pas de le dire, puisque l'apport de pollen non viable ou la castration elle-même peuvent stimuler la parthénocarpie), DOVEDAR a réalisé des croisements entre du matériel mâlestérile ( Pearson $m s »$ ) ou «stamenless», d'une part, et «Severianin», d'autre part. Dans les descendances, il a observé la formation de fruits parthénocarpiques chez des plantes mâle-stériles ou "stamenless »; c'est donc que la parthénocarpie chez «Severianin » est non stimulative.

DOVEDAR a montré que le pollen de «Severianin » est viable (ce pollen a été testé sur «Belij Naliv 241 ", sur «Pearson $m s »$ et sur un mutant «stamenless »). DoveDAR a, par ailleurs, fait une étude cytologique comparée de la germination du pollen et des premières étapes de la fructification, chez «Severianin», parthénocarpique, et chez "Vinequeen", non parthénocarpique. Les études ont été faites sur des fleurs castrées pollinisées avec le pollen de la même variété. Les ovaires pollinisés ont été récoltés à intervalles réguliers, de 3 à $96 \mathrm{~h}$ après la pollinisation. $\mathrm{Chez}$ "Vinequeen", les tubes polliniques atteignent les sacs embryonnaires $18 \mathrm{~h}$ après la pollinisation; la fécondation est assurée $24 \mathrm{~h}$ après la pollinisation. Chez « Severianin », $12 \mathrm{~h}$ après la pollinisation, les extrémités des tubes polliniques commencent à grossir et à se replier; $36 \mathrm{~h}$ après la pollinisation, seuls quelques tubes polliniques ayant atteint les sacs embryonnaires assurent la fécondation. Ce comportement des tubes polliniques de "Severianin ", dont l'extrémité s'épaissit et se replic et dont la croissance est ralentie, font conclure à Dovedar (1973), AVAKIMOVA \& DoveDAR (1976), TARAKANOv et al. (1978) que la formation de fruits sans graines chez cette variété est due à une autoincompatibilité, la croissance des tubes polliniques étant inhibéc dans le tissu conducteur du style. Dovedar (1973) a par ailleurs montré que la germination in vitro du pollen de "Severianin " était fortement inhibée quand ce pollen était en présence d'extraits de styles ou d'extraits d'ovaires

TABLEAU 3

Influence de différents traitements sur la nouaison, le poids moyen du fruit et le nombre de graines par fruit chez "Severianin» (d'après DOVEDAR, 1973).

Effect of different treatments on fruit-set, average weight of fruits and number of seeds per fruit, in "Severianin " (after DOVEDAR, 1973).

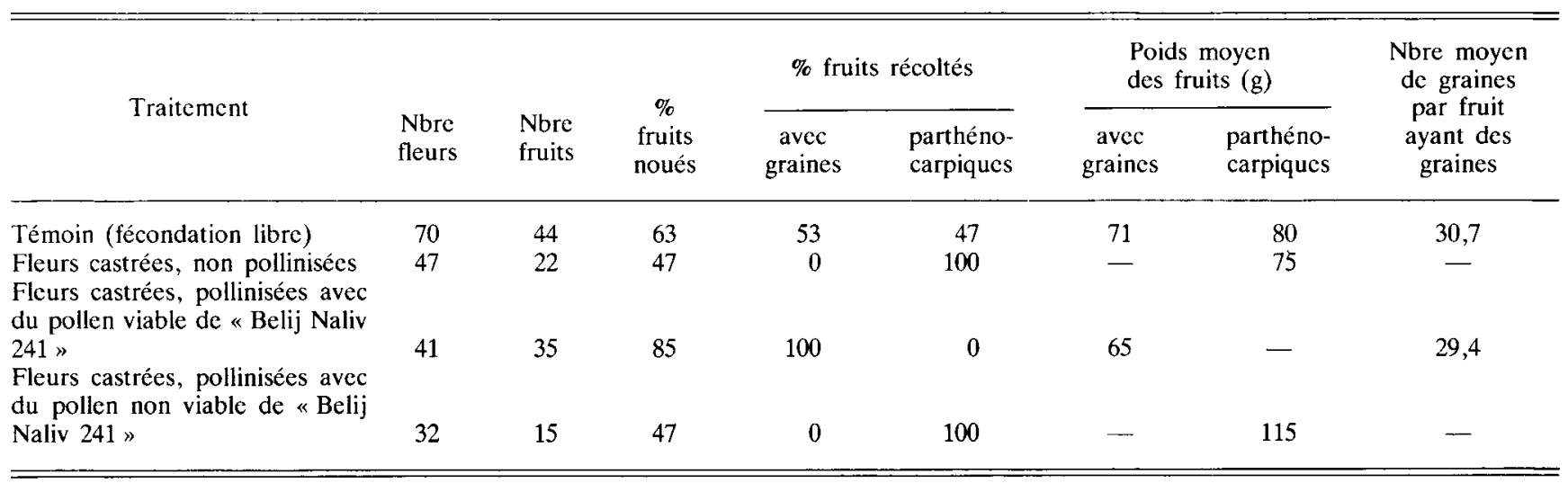


( $22 \mathrm{~h}$ après la pollinisation) issus de fleurs pollinisées de «Severianin »; la germination du pollen de «Belij Naliv 241 » était au contraire stimulée en présence de tels extraits.

Dovedar (1973), AVakimova \& Dovedar (1976) ont étudié la teneur des ovaires en acides aminés libres chez «Severianin» et «Vinequeen", sur des fleurs préalablement castrées et pollinisées avec du pollen de la même variété, et chez "Severianin » sur des fleurs castrées et non pollinisées ; les ovaires ont été récoltés à intervalles réguliers entre 6 et $96 \mathrm{~h}$ après la pollinisation. Chez les 2 variétés, l'accumulation des acides aminés libres dans les ovaires se fait surtout dans les 36 premières heures et leur teneur devient beaucoup plus grande que dans les ovaires issus de fleurs castrées et non pollinisées de «Severianin ». Au moment de la fécondation, il y a plus d'acides aminés chez "Vinequeen" que chez «Severianin». Dans les ovaires de "Severianin », il y a accumulation d'une grande quantité de phénylalanine, alors qu'on n'en trouve pas chez «Vinequeen ».

DOvedar (1973) a étudié le déterminisme génétique de la parthénocarpic de «Severianin » sur la descendance du croisement «Belij Naliv $241 » \times$ « Severianin ». Le caractère est dû à un seul gène récessif (sur 107 plantes F2, 33 étaient parthénocarpiques et 74 non parthénocarpiques).

\section{Travaux entrepris dans d'autres pays}

En France, la variété «Severianin» a été étudiée à la Station d'Amélioration des Plantes maraîchères de l'I.N.R.A., à Montfavet. Cette variété, cultivée sous serre verre non chauffée au cours du printemps 1976, a montré son aptitude à développer des fruits parthénocarpiques en conditions de température basse (PHILOUZE \& MAISONNEUVE, 1978c). A la suite de ces premières observations, des essais ont été entrepris, qui ont montré que "Severianin " était capable, après castration des fleurs sans pollinisation, de développer des fruits sans graines de même aspect et de même grosseur que les fruits avec graines obtenus après castration et pollinisation avec du pollen de «Severianin » ou d'une autre variété non parthénocarpique.

Cette parthénocarpie n'est pas due à une stérilité mâle ou femelle, puisqu'il est possible d'obtenir des graines par autofécondation (soit autofécondation naturelle, soit autofécondation manuclle). A cet égard, le terme d'auto-incompatibilité cmployé par les auteurs russes nous paraît tout à fait impropre. A notre avis, l'arrêt dle croissance des tubes polliniques observé par ces auteurs est vraisemblablement dû au fait que l'ovaire commençant sa croissance avant la floraison, les tubes polliniques sont dans l'impossibilité d'assurer la fécondation des ovules qui ne sont plus réceptifs.

L'étude de l'hérédité du caractère a été faite avant que nous ayons eu connaissance du travail de DOVEDAR. Nous avons attribué le symbole pat-2 au gène récessif responsable de ce caractère (PhILOUzE \& MAISONneuve, 1978a); le symbole $p$, proposé par DovEDAR (1973) ne pouvait être retenu, étant déjà utilisé pour désigner un autre mutant, «peach ». Nous avons montré que le gène responsable de la parthénocarpie chez «Severianin » était situé à un locus différent de cclui de pat décrit par SORESSI \& SALAMINI (1975).

L'expression du caractère de parthénocarpie dépend du milieu (Philouze \& Maisonneuve, 1978a; Philouze, 1981). Ainsi, si au printemps et en été les fruits sans graines de «Severianin » sont de même grosseur que les fruits avec graines, il n'en est pas de même pour des fruits issus de fleurs épanouies en fin septembre-octobre, castrées et non pollinisées. Le pourcentage de fruits noués à partir de ces fleurs et le poids moyen des fruits sont plus faibles que dans le cas de fruits avec graines issus de fleurs épanouies aux mêmes dates.

Un travail de sélection avec le gène pat-2 a été entrepris, afin de diversifier le matériel parthénocarpique. Les premières observations sur ce matériel ont montré que l'expression du caractère de parthénocarpie dépendait également du génotype (PHILouze, 1981). Enfin, la création de matériel isogénique, sauf pour pat-2, est en cours; ce matériel est indispensable pour étudier de façon rigoureuse l'effet du gène pat-2 sur une série de caractères agronomiques, physiologiques et biochimiques.

A la suite des travaux conduits en U.R.S.S. et en France avec la variété "Severianin ", un grand intérêt s'est manifesté dans plusieurs pays pour l'aptitude à la parthénocarpie naturelle de cette variété. Citons les travaux entrepris aux Pays-Bas par VAN DEN BERKMORTEL (1978), HOGENBOOM (1978 à 1982), ZIJLSTRA (1979), en Italie par PORCELli et al. (1979), PORCELLI \& SORESSI (1981), aux Etats-Unis par LIN (1982), LiN et al. (1982), GEORGE et al. (1982). Ces auteurs américains ont montré, après Dovedar (1973), que « Severianin " était capable de développer des fruits parthénocarpiques à température élevée.

\section{DISCUSSION ET CONCLUSION}

L'aptitude à la parthénocarpie naturelle chez la tomate fait l'objet depuis quelques années de nombreux travaux. Jusqu'à une date récente, ces travaux ont été conduits de façon indépendante et il devenait nécessaire de tester, dans les mêmes conditions, le matériel connu dans la littérature pour son aptitude à la parthénocarpie. Cela a été réalisé dans plusieurs pays, avec le souci de rechercher également de nouveaux géniteurs de parthénocarpie. Ainsi aux PaysBas, ce travail a commencé, en été 1977 (ZiJlsTrA, 1979), par l'étude d'une série de variétés citées dans la littérature ainsi que du matériel sélectionné à Wageningen ; les fleurs ont été castrées, les unes ont été pollinisées, les autres non. Parmi la centaine de varićtés et de lignées étudiées, une aptitude à la parthénocarpic a été trouvée chez une trentaine de lots (HOGENBOOM, 1978, 1979, 1980) ; cependant «Severianin » semble l'origine lá plus intéressante.

De même en Pologne, PASZKOWSKA (1982) a testé 30 variétés de tomate pour leur aptitude à la parthénocarpie. Plusicurs critères, tels que le pourcentage de fruits parthénocarpiques ou le pourcentage de nouaison à partir de fleurs castrées, ont été utilisés. En fait, l'aptitude des différentes variétés est variable. Cependant, la parthénocarpie de «Early North» et de «Parteno», comme celle de "Lyconorma " et «Lycoprea », est bien marquée et serait monogénique récessive.

Les sources connues de parthénocarpie viennent soit de mutations à des loci individualisables (pat; pat-2), soit d'un lent travail de cumul de gènes favorables; cela semble le cas pour le matéricl obtenu en Allemagne à partir du croiscment « Atom» $x$ «Bubjekosoko ». De même, KUBICKI \& MiCHALSKA (1978), en Pologne, - à partir d'un croisement entre les 2 variétés canadiennes "Early North" (qui montre une tendance limitée à développer des fruits sans graines) et «Beaverlodge 6703» (qui ne montre pas une telie tendance) - ont sélectionné des lignées qui ont une tendance marquée à former des fruits parthénocarpiques quand les conditions du milieu sont défavorables à la fécondation ; ce 
caractère résulterait d'une combinaison de facteurs génétiques venant des 2 variétés parentales.

La prospection de la descendance de croisements interspécifiques peut se révéler intéressante. LESLEY \& LESLEY (1953) ont attribué à une combinaison exceptionnelle de gènes venant de 2 espèces (combinaison qui entraînerait une production excessive d'auxine) plutôt qu'à l'état tétraploïde lui-même, l'aptitude très marquée à la parthénocarpie (avec grossissement de l'ovaire avant l'ouverture de la fleur) que montrait une plante tétraploïde, mâle et femelle stérile, issue d'une plante F1 tétraploïde provenant du croisement $L$. esculentum $\times L$. peruvianum var. dentatum, suivi de 2 recroisements sur une plante tétraploïde à petits fruits de L. esculentum. Cette hypothèse est basée sur le fait qu'on ne trouve pas une telle aptitude à là parthénocarpie dans des lignées sœurs tétraploïdes, pas plus que dans les plantes parentes utilisées pour la réalisation du $2^{\mathrm{c}}$ recroisement. D'autre part, LESLEY \& LESLEY auraient également obtenu des plantes parthénocarpiques, bien que d'un niveau de parthénocarpie moindre, chez des plantes diploïdes issues d'un croisement $L$. esculentum $\times L$. hirsutum.

Aux Pays-Bas, une source prometteuse de parthénocarpie a été trouvée dans un croisement interspécifique avec $L$. peruvianum (HOGENBOOM, 1980, 1981). D'autre part, une nouvelle origine de parthénocarpie a été trouvée dans le porte-greffe « $\mathrm{KK}$ », hybride interspécifique avec $L$. hirsutum. (HogenboOM, 1980). En France, du matériel parthénocarpique a été obtenu dans la descendance d'un croisement avec L. parviflorum (PHILOUZE, non publié). Enfin, rappelons que «Severianin» est issue d'un croisement avec L. hirsutum.

Jusqu'à présent, la seule distinction que nous ayons introduite pour présenter les cas de parthénocarpie connus chez la tomate est entre parthénocarpie artificielle, qui en fait ne nous intéresse pas ici, et parthénocarpie naturelle. Une distinction supplémentaire mérite d'être introduite entre parthénocarpic naturelle accidentelle (ou occasion- nelle) et parthénocarpie naturelle facultative, bien que, toute une gamme de manifestations intermédiaires existant, il puisse être difficile de rattacher tel ou tel exemple à un groupe plutôt qu'à un autre. Pour simplifier, nous définirons comme parthénocarpie accidentelle les cas où la formation de fruits parthénocarpiques est peu fréquente et où ces fruits sans graines sont en général d'aspect sensiblement différent de celui des fruits avec graines (plus petits, plus côtelés, plus ternes) et plus lents à mûrir. En l'absence d'études physiologiques approfondies, nous pouvons seulement faire l'hypothèse que, dans les cas de parthénocarpie accidentelle, les facteurs du milieu agiraient de façon prépondérante sur les facteurs génétiques, en entravant la pollinisation et la fécondation. Beaucoup des exemples cités en $1^{\text {ère }}$ partie peuvent vraisemblablement être rattachés à ce groupe; citons en particulier les exemples donnés par HÖSTERMANN $(1912,1913)$ et par OSBORNE \& WENT (1953).

Nous définirons comme parthénocarpie facultative les cas où la formation de fruits parthénocarpiques est relativement fréquente et peut même atteindre 100 p. 100 des fruits formés dans certaines conditions. Ces fruits sans graines sont d'aspect et de taille comparables, sinon identiques, aux fruits avec graines; lcur précocité est également comparable. Nous faisons l'hypothèse que, dans ce cas, les facteurs génétiques agiraient de façon prépondérante sur les facteurs du milieu, en favorisant la production d'auxines dans les ovaires, donc en permettant à ceux-ci de se développer sans aucun retard et même avant la floraison. La parthénocarpie de «Severianin », contrôlée par le gène pat-2, est un exemple de parthénocarpie facultative.

En définitive, les cas de parthénocarpic les plus intéressants chez la tomate relèvent d'une parthénocarpie naturelle facultative; celle-ci est d'origine génétique, mais son expression dépend aussi des facteurs du milieu.

Reçu le 6 décembre 1982. Accepté le 2 mars 1983.

\section{RÉFÉRENCES BIBLIOGRAPHIQUES}

Allavena A., Soressi G. P., 1978. Tetraploid parthenocarpic fruitful tomatoes. Rep. Tomato Genet. Coop., 28, 2.

Avakimova L. G., Dovedar S. A., 1976. The content of free amino acids in tomato fruitlets after self compatible and self incompatible pollination. (en russe) Izv. Timiryazevskoi Sel'sko-kh. Akad., 6, 127-134.

Baggett J. R., Frazier W. A., 1978a. Orcgon Cherry tomato. HortScience, 13 (5), 598.

Baggett J. R., Frazier W. A., 1978b. Oregon T 5-4 parthenocarpic tomito line. HortScience, 13 (5), 599.

Bianchi A., Soressi G. P., 1969. Mutanti di pomodoro artificialmente indotti suscettibili di utilizzazione nel miglioramento genetico. Sementi Elette, 15 (3), 194-198.

Dovedar S. A., 1973. Etudes des particularités de la mise à fruit des tomates soumises à des températures élevées. Mise au point de méthodes pour améliorer cette mise à fruit (en russe). Résumé de thèse présentée à l'Académie d'Agriculture Timiriazev de Moscou, $16 \mathrm{p}$.

El Ahmadi A. B., Stèvens M. A., 1979. Genetics of high temperature fruit set in the tomato. J. am. Soc. Hort. Sci., 104 (5), 691-696.

Falavigna A., Badino M., Soressi G. P., 1978. Potential of the monomendelian factor pat in the tomato breeding for industry. Genet. Agrar., 32 (1/2), 160 (Abstr.).'

Foster A. C., Tatman E. C., 1937. Environmental conditions influencing the development of tomato pockets or puffs. $\mathrm{Pl}$. Physiol., 12, 875-880. (cité par Gustafson, 1942).
George W. L., Lin S., Splittstoesser W. E., 1982. Breeding tomatocs for improved fruit setting under environmental stress using parthenocarpy. 21st Int. Hort. Cong. Hamburg, Fed. Repub. of Germany, August-September 1982, 1447 (Abstr.).

Gustafson F. G., 1936. Inducement of fruit development by growthpromoting chemicals. Proc. natl. Acad. Sci., 22, 628-636.

Gustafson F. G., 1939. The cause of natural parthenocarpy. Am.J. Bot., 26, 135-138.

Gustafson F. G., 1942. Parthenocarpy : natural and artificial. Bot. Rev., 8, 599-654.

Harris R. E., 1972. Three new sub-arctic type tomatoes : « Early Sub-Arctic», "Sub-Arctic Midi», and «Sub-Arctic Plenty». Can. J. Plant Sci., 52, 119-120.

Hawthorn L. R., 1937. Seedlessness in tomatoes. Science, 85, 199. Hogenboom N., 1978. Instituut voor de Veredeling van Tuinbouwgewassen (IVT). Annu. Rep. 1977, 22.

Hogenboom N., 1979. IVT. Annu. Rep. 1978, 21-22.

Hogenboom N., 1980. IVT. Annu. Rep. 1979, 29-30.

Hogenboom N., 1981. IVT. Annu. Rep. 1980, 26-27.

Hogenboom N., 1982. IVT. Annu. Rep. 1981, 29.

Höstermann G., 1912-1913. Berichte über die Tätigkeit der wissenschaftlichen Institute. I. Pflanzenphysiologische Versuchsstation. Ber. Kgl. Gartenl., Dahlem., 85-106, 1912/54-62, 1913 (cité par Gustafson, 1942).

Johnson S. P., Hall W. C., 1954. Parthenocarpy in the tomato. Proc. am. Soc. Hort. Sci., 63, 329-332. 
Kraevoj I. M., 1949. Création d'une nouvelle forme de tomate par hybridation sexuée et végétative (en russe) Agrobiologiya., 4, 109 115.

Kubicki B., Michalska A. M., 1978. Transgression of early yield in hybrids of early forms of tomato (Lycopersicon esculentum Mill.). Genetica Polonica, 19 (3), 291-306.

Lesley J. W., Lesley M. M., 1953. Fruitfulness, fertility and parthenocarpy in tetraploid species hybrids of Lycopersicon. J. Hered., 44, 261-264.

Lesley M. M., Lesiley J. W., 1941. Parthenocarpy in a tomato deficient for a part of a chromosome and in its aneuploid progeny. Genetics, 26, 374-386.

Lin S., 1982. The genetic, breeding and physiology of parthenocarpy in Lycopersicon esculentum Mill. (tomato). Diss. Abstr. Int. B., $42(9), 3514$ B.

Lin S., George W. L., Splittstoesser W. E., 1982. Parthenocarpy and the ability to set fruit under high temperature conditions. Rep. Tomato Genet. Coop., 32, 35-36.

Ludnikova L., 1970. Parthenocarpie chez la tomate (en russe). Kartja Moldovenjaske, Kishthev, Motaavian SSR, 98 p.

Luneva M. Z., 1957. Une nouvelle forme de tomate (en russe). Sel'khozgiz (cité par Ludnikova, 1970).

Mapelli S., 1981. Changes in cytokinin in the fruits of parthenocarpic and normal tomatoes. Plant Sci. Leti., 22, 227-233.

Mapelli S., Frova C., Torti G., Soressi G. P., 1978. Relationship between set, development and activities of growth regulators in tomato fruits. Plant Cell. Physiol., 19 (7), 1281-1288.

Mapelli S., Torti G., Badino M., Soressi G. P., 1979. Effects of GA3 on flowering and fruit set in a mutant of tomato. HortScience, 14 (6), 736-737

Musehold J., 1972. Untersuchungen über den Zusammenhang von spontanen Änderungen im Grad der Parthenokarpic und dem natürlichen Wuchsstoffgehalt bei drei Genotypen von Tomaten. Garte:tbauwissenschaft, 37 (4), 281-292.

Nitsch J. P., 1952. Plant hormones in the development of fruits. The Q. Rev. Biol., 27, 33-57.

Nitsch J. P., 1970. Hormonal factors in growth and development. in Food Science and Technology. A series of Monographs. Ed. Hulme AC, vol. 1, Ac. Press, London, 427-472.

Noll F., 1902. Fruchtbildung ohne vorausgegangene Bestäubung (Parthenokarpic) bei der Gurke. Sitzungsb. Niederrhein. Ges. Nat. Heilk. Bonn, 149-162 (cité par Gustafson, 1942).

Osborne D. J., Went F.W., 1953. Climate factors influencing parthenocarpy and normal fruit-set in tomatocs. Bot. Gaz., 114, 312-322.

Paszkowska I., 1982. Occurrence of parthenocarpy in self pruning tomato cultivars. 21st Int. Hort. Congr., Hamburg, Fed. Repub. of Germany, August-September 1982, 1446 (Abstr.).

Pecaut P., Philouze J., 1978. A sha pat line obtained by natural mutation. Rep. Tomato Genet. Coop., 28, 12.

Philouze J., 1981. Etat des travaux sur l'utilisation en sélection de l'aptitude à la parthénocarpic naturelle de la variété de tomate "Scverianin" in Gcnetics and Brceding of Tomato. Proc. of the Meeting Eucarpia Tomato Working Group, Avignon, France, May 1981, 203-210.

Philouze J., Maisonneuve B., 1978a. Hcredity of the natural ability to set parthenocarpic fruits in the Soviet varicty "Severianin". Rep. Tomato Genet. Coop., 28, 12-13.

Philouze J., Maisonneuve B., 1978b. Heredity of the natural ability to set parthenocarpic fruits in a German line. Rep. Tomato Genet. Coop., 28, 12.

Philouze J., Maisonneuve B., 1978c. Breeding tomatoes for their ability to set fruit at low temperatures. In Genotype and environment in glasshouse tomato breeding. Proc. Meeting Eucarpia Tomato Working Group, Leningrad, USSR, May 1978, 54-64.

Porcelli S., Soressi G. P., 1981. Miglioramento genetico delle ortive per coltura protetta. Colture protette, 10 (2), 19-22.

Porcelli S., Soressi G. P., Uncini L., 1979. Miglioramento genetico del pomodoro da mensa. Colture protette, 8 (7), 19-24.

Preil W., 1973. Zur Parthenokarpie bei Tomaten in Abhängigkeit vom Temperaturverlauf. Angew. Botanik, 47, 135-140.

Preil W., 1978. Zuchtziele bei Tomaten. Gemüse, 14 (2), 48-51.

Preil W., Reimann-Philipp R., 1969. Untersuchungen über die Einflüsse verschiedener Umweltfaktoren auf die Funktionsfähigkeit der Pollen von Tomaten. Angew. Bot., 43, 175-193.

Reimann-Philipp R., 1968. Die Ausnutzung des Merkmals « Parthenokarpic » zur Züchtung extrem frühreifer Sorten bei der Tomatc. In Arbeiten der Abteilung « Höhere Pflanzen ». Vorträge gehalten am 4-12-1968 im Max-Planck-Institut für Kulturpflanzenzüchtung, Hamburg, 15-36.

Reimann-Philipp R., Fuchs G., 1969. Verfrühung der Tomatenernte unter Glas durch Verwendung von Zusatzlicht und speziell selektierten Genotypen Buschtomaten. Erwerbsgärtner, 23, 2153 2155.

Richter E., 1972. Der Gchalt an geschmackgebenden Inhaltsstoffen von parthenokarpen und samenhaltigen Tomaten. Gartenbauwissenschaft., 37 (3), 201-211.

Rybchenko O. I., 1959. The development of ovules in parthenocarpic forms of tomatoes (en ukrainien). J. Bot. Acad. Sci. Ukr., 16 (1) (cité par Ludnikova, 1970).

Rybchenko O. I., 1960. Cytoembryologie du développement des fruits parthénocarpiques chez les tomates (en russe). Kand. Diss. (cité par Ludnikova, 1970).

Soressi G. P., 1970. Tomato mutants following EMS treatments. Rep. Tomato Genet. Coop., 20, 59.

Soressi G. P., Salamini F., 1975. A monomendelian gene inducing parthenocarpic fruits. Rep. Tomato Genet. Coop., 25, 22.

Stout A. B., 1936. Seedlessness in grapes. N.Y. State Agric. Exp. Sta. Tech. Bull., 238 (cité par Gustafson, 1942).

Tarakanov G. I., Dovedar S. A., Avakimova L. G., Andreeva E. N., Sisina E. A., 1978. The ways to increase fruit-setting in tomatoes under high temperatures (en russe). In Genotype and cnvironment in glasshouse tomato breeding. Proc. Meeting Eucarpia Tomato Working Group, Leningrad, USSR, May 1978, 123-129.

Tatarintsev A. S., 1929. Parthenocarpy in tomatoes (en russe) Izv., Sel'sk.-Khoz. Akad. K. A. Timiryazeva, 4, 125-141.

Ulrich R., 1952. Nouaison sans fécondation préalable. Parthénocarpie. La vie des fruits, Masson et Cie Ed., 28-34.

Van den Berkmortel L. G., 1978. Breeding greenhouse tomatoes with lower energy requirement. In " Genotype and environment in glasshouse tomato breeding". Proc. Meeting Eucarpia Tomato Working Group, Leningrad, USSR, May 1978, 31-35.

Vazart B., 1955. La parthénocarpie. Bull. Soc. Bot. Fr., 102 (7/8), 406-443.

Winkler H., 1908. Über Parthenogenesis und Apogamic im Pflanzcnreichc. Prog. Rei. Bot. 2, 293-454 (cité par Gustafson, 1942). Zijlstra S., 1979. Secdless tomatoes : a new perspective ? (en hollandais). Tuinderij., 19 (2), 104-105. 